\title{
Diversidad y mecanismos de dispersión de árboles de la Isla Gorgona y de los bosques húmedos tropicales del Pacífico colombo-ecuatoriano
}

\author{
Roxana Yockteng y Jaime Cavelier ${ }^{1}$ \\ Departamento de Ciencias Biológicas, Universidad de los Andes, AA 4976, Bogotá, Colombia FAX 57-1-281890. Internet: \\ jcavelie@uniandes.edu.co yr-yockte@uniandes.edu.co
}

\section{Recibido 24-VII-1996. Corregido 24-IX-1997. Aceptado 09-X-1997}

\begin{abstract}
The flora of the continental Island of Gorgona (Colombia) and that of the forests on the Chocó biogeographical region of Colombia and Ecuador were compared with species-area curves and species diversity indexes, either previously published or calculated using the original data of the vegetation surveys. The curves and the Shannon diversity index showed that the forest of Gorgona is less rich in tree species than the mainland sites. Furthermore, the Shannon equitability index in five continental lowland forests was higherthat in Gorgona, suggesting that the number of trees per species is more evenly distributed in the continental forests. In Gorgona, seed and fruit dispersal was primarily carried out by birds ( $49.7 \%$ of the species), as reported for the islands of Galapagos in Ecuador (60\%) and in the biogeographic island of the cloud forest of the Serranía de Macuira, Guajira, in the north of Colombia $(51 \%)$. In contrast, dispersal by mammals was the main mechanism for tree species in the continental forests of Cabo Corrientes, Bajo Calima (Colombia) and Los Tuxtlas (México). Although the forest at Gorgona is not particularly rich on tree species, it plays an important role in the protection of marine ecosystems. A reduction in forest cover would result in an increase in soil erosion rates and landslides that would greatly affect marine biota, particularly of coral reefs.
\end{abstract}

Key words: Species richness, diversity, dispersal, lowland rain forest, island, Chocó.

Existe una relación directa entre el área de una isla y el número de especies de plantasy animales que puede soportar. Esta relación puede ser explicada en términos de un mayor número de hábitats en áreas grandes que en pequeñas (Lack 1969, 1976, Williamson 1981)ocomo un balance entre las tasas de inmigración y extinción (MacArthur \& Wilson 1967). Sin embargo, esta relación especies-área puede ser distorsionada por la presencia de endemismos (Quinn \& Harrison 1988). En las Islas Galápagos, por ejemplo, el número de especies de plantas de la isla de Isabela ( 344 especies) es menor que en el de una serie de islas con un área combinada igual a la de esta isla (609 especies). En el caso de islas que estuvieron unidas al continente, es posible observar una disminución del número de especies que soporta la isla a medida que aumenta el tiempo de separación del continente, fenómeno conocido como "relajación" (Wilcox 1978).

$\mathrm{La}$ isla Gorgona es considerada por algunos autores como el último remanente en la costa pacífica colombiana de una cordillera que nace en la Serranía del Baudó y la Serranía de los Saltos (Gansser 1950, Nygren 1950, Haffer 1970). Durante el Pleistoceno, el nivel del mar bajó aproximadamente 120 metros, lo cual permitióla unión entre la isla Gorgona y el continente (Alberico 1986). Estauniónexplicaría la similitud entre la flora y la fauna de la costa y la isla, similitud que sería más fuerte con el sur (Tumaco, Nariño) que con el norte de la costa pacífica (Alberico 1986, Lourenco \& Flores 1989). 
El número de especies en una isla puede ser comparado con el de un área similar en el continente haciendo uso de las curvas de acumulación de especies en función del área de muestreo. Por lo general, el número de especies en un área del continente es significativamente mayor que para una islade igual tamaño(Richman et al. 1988). Esta diferencia ha sido interpretada como el resultado de tamaños poblacionales menores en la isla y por lo tanto mayores tasas de extinción, asícomopor la facilidad de inmigración y emigración en el área del continente que se compara con la isla, físicamente aislada de la fuente de especies.

El análisis de la fauna y flora de las islas y sus diferencias con el continente también pueden ser analizadas desde la perspectiva de los mecanismos de dispersión de las diásporas. En las plantas en particular, las características morfológicas y anatómicas de las semillas pueden ser indicadoras de diferentes "síndromes" de dispersión (Howe \& Westley 1988). Por ejemplo, las semillas dispersadas porvientoson livianas y de superficie grande, mientras que las semillas dispersadas por aves pueden tener arilos de colores llamativos (Renner 1987, Howe \& Westley 1988). Enelcaso de islas lejanas y pequeñas, sería de esperar un mayornúmerode especies de plantas dispersadas por agua y aves que por mamíferos grandes (Howe 1984).

El presente trabajo es una comparación entre la flora arbórea de la Isla de Gorgona y de los bosques del Pacíficocolombo-ecuatorianodesde la perspectiva de la biogeografía de islas y de los mecanismos de dispersión de semillas. Más específicamente, se quiere dar respuesta a las siguientes preguntas: 1) ¿Cómo se comparan la riqueza y diversidad de especies de árboles entre la Isla Gorgona y las tierras bajas del continente? 2) ¿Cómosecomparan los síndromes de dispersión de semillas del bosque de la isla y uno similar en el continente?

\section{MATERIALES Y MÉTODOS}

Areas de estudio: La isla Gorgona $\left(2^{\circ} 58^{\prime} \mathrm{N}\right.$; $78^{\circ} 11^{\prime} \mathrm{W}$ ) está situada a $56 \mathrm{~km}$ de la costa del DepartamentodelCauca sobreelOcéanoPacífico, Colombia (Prahl et al. 1979). Es una isla continental conunárea de 1569 hectáreas, cubierta de bosque muy húmedo tropical (Espinal \& Montenegro 1977). Desde el punto de vista florístico está cubierta por tres asociaciones de bosques naturales, zonas de cultivos; vegetación secundaria,comunidades pioneras sobre playas y fases subseriales (Rangel \& Aguirre 1993, Rangel 1990). En el flanco occidental de la isla se desarrollan importantes ecosistemas de coral (Prahl et al. 1979). La isla tiene una precipitación media anual de $6694 \mathrm{~mm}$ (Rangel \& Aguirre 1993) y una temperatura media anual de $27^{\circ} \mathrm{C}$ (Prahl et al. 1979). No se presentan meses con menos de $100 \mathrm{~mm}$ de precipitación y la humedad relativa es alta y constante $(90 \%)$.

Para realizar la comparación de la flora de la isla Gorgona con la del Pacífico, se seleccionaron dos localidades; Cabo Corrientes (Chocó) y Bajo Calima (Valle del Cauca). Cabo Corrientes está situado en la costa pacífica del Departamento del Chocó $\left(5^{\circ} 37^{\prime} \mathrm{N} ; 77^{\circ} 30^{\prime} \mathrm{W}\right)$. Estelugartiene una precipitación media anual superior a los 5000 $\mathrm{mm}$ y corresponde a un bosque muy húmedo tropical (bmh-T). El área en general está cubierta por un bosque maduro denso, del cual se han extraído algunos árboles de maderas valiosas (Galeano etal. 1993). Bajo Calima estásituadaen el sur de la región del río San Juan y del río Calima en el Departamento del Valle del Cauca $\left(3^{\circ} 55^{\prime} \mathrm{N}\right.$, $\left.77^{\circ} 00^{\prime} \mathrm{W}\right)$. Esta zona tiene una precipitación media anual de $7000 \mathrm{~mm}$ y está clasificada como un bosque muy húmedo tropical (bmh-T). La media de la temperatura anual es $27.3^{\circ} \mathrm{C}$ (FaberLangendoen \& Gentry 1991). Los bosques de la Isla Gorgona también se compararon con dos bosques húmedos tropicales en Río Palenque, en el flanco occidental de los Andes ecuatorianos (2 $980 \mathrm{~mm}$; Gentry 1986), con un bosque húmedo tropical en el Parque Nacional Natural Los Katíos en el Darién colombiano (1 200 mm; Floresta 1984) y con un bosque de manglares en Satinga, 
sobre la costa pacífica colombiana en frente a la Isla Gorgona (Codemaco 1973).

Censos de vegetación: La información sobre la composición florística de los bosques de la isla Gorgona fue obtenida de 15 levantamientos florísticos de $500 \mathrm{~m}^{2}(20 \mathrm{~m} \times 25 \mathrm{~m})$ distribuidos al azaren los terrenos de la Isla cubiertos por bosques maduros (Rangel 1990). Para el presente trabajo, sólo se utilizaron los datos de árboles con una altura mayor de $5 \mathrm{~m}$, aproximadamente equivalente a árboles con un diámetro a la altura del pecho $>10 \mathrm{~cm}$. El inventario florístico realizado por Galeano et al. (1993) en Cabo Corrientes, tuvo una metodología similar a la utilizada en la isla Gorgona. En Cabo Corrientes, se realizaron 10 parcelas de $1000 \mathrm{~m}^{2}$ donde se muestrearon los árboles de diámetro mayor a 10 $\mathrm{cm}$. En Bajo Calima, el censo de vegetación se realizó en una parcela de $10000 \mathrm{~m}^{2}$ y se recolectaron muestras botánicas de árboles con un diámetro a la altura del pecho (d.a.p.) mayor a $10 \mathrm{~cm}$ (Faber-Langendoen \& Gentry 1991).

Curvas de acumulación de especies: Se realizaron curvas de acumulación de especies en función del área muestreada, utilizando la información de los censos de vegetación de la Isla Gorgona(Rangel 1990). Las curvas de los bosques de la región de Bajo Calima (Faber-Langendoen \& Gentry 1991) y del bosque de Cabo Corrientes (Galeano et al. 1993), fueron realizados por los investigadores que hicieron los censos. También se generaron las curvas de acumulación de especies con base en los censos de vegetación realizados en 20 parcelas de $500 \mathrm{~m}^{2}$ en los manglares de Satinga,Nariño(Codemaco 1973) y en l0parcelas de $1000 \mathrm{~m}^{2}$ en el bosque del Parque Nacional Natural los Katíos, Chocó (Floresta 1984). En amboscasos se midieron losárboles de más de 10 cm de d.a.p.

Indicesdediversidad: Con base en los listados de especies de la isla Gorgona, de CaboCorrientes y de Bajo Calima se aplicaron los índices de diversidad de Shannon (H) y Simpson (D) y los índices de equitabilidad deShannon $(\mathrm{J})$ y Simpson (E) (Begon et al. 1996, Magurran 1988). Estos valores se compararon con los de los bosques de Río Palenque 1 y 2 en Ecuador y Tutunendó en Colombia (Alvarez, 1993). Los valores de los índices de equitabilidad de Shannon y de Simpson varían entre 0 y 1 donde 0 representa la máxima dominancia y 1 la máxima diversidad (Begon et al. 1996, Magurran 1988).

Mecanismos de dispersión de frutos y semillas: Seestudiaronlosdiferentesmecanismos de dispersión de frutos y semillas (dispersión por aves, mamíferos, murciélagos, viento y agua) para las especies de la isla y el continente, utilizando los criterios descritos en Burrows (1986), Renner (1987) y Howe \& Westley (1988). Las características de los frutos y de semillas que fueron utilizados para la definición de los mecanismos de dispersión fueron obtenidas de Valle (1973), Bartholomäus etal. (1990), Gentry (1993), Sugden (1982), Auspurger(1986), Foster etal. (1986), Zona \& Henderson (1989), Blake \& Loiselle (1992) y Mori \& Brown (1994). Para compararestos resultados con los de otros bosques, se determinaron en la misma forma los mecanismos de dispersión de las especies del ParqueLos Tuxtlasen México (Ibarra-Manríquez \& Oyama 1992). Los porcentajes de árboles dispersadospordiferentesmecanismos(p.ej.aves, mamíferos) fueron calculados con base enl número de especies a las cuales se les pudo asignar un dispersor. El porcentaje de árboles a los cuales no se les pudo asignar dispersor varió de $7 \%$ (Isla Gorgona)a 24\% (Bajo Calima). Paracada bosque y en caso de que una especie tuviera más de un dispersor, se le asignaba un valor proporcional al número de dispersores (Un dispersor $=1.0$, dos dispersores $=0.5$, tres dispersores $=0.33$ ).

\section{RESULTADOS}

Curvas de acumulación de especies: De las cinco curvas de acumulación de especies, la del bosque de Bajo Calima fue la más alta, seguida de la de Cabo Corrientes, Isla Gorgona, Parque Los Katíos y manglares de Satinga. (Fig. 1). Con excepción del bosque de Bajo Calima, en todos 
los otros bosques se obtuvo una saturación de la curva especies-área. Esto indica que el área de muestreo es suficiente para medir la riqueza de especies en la mayoría de estos bosques (Connor $\&$ Mc Coy 1979).

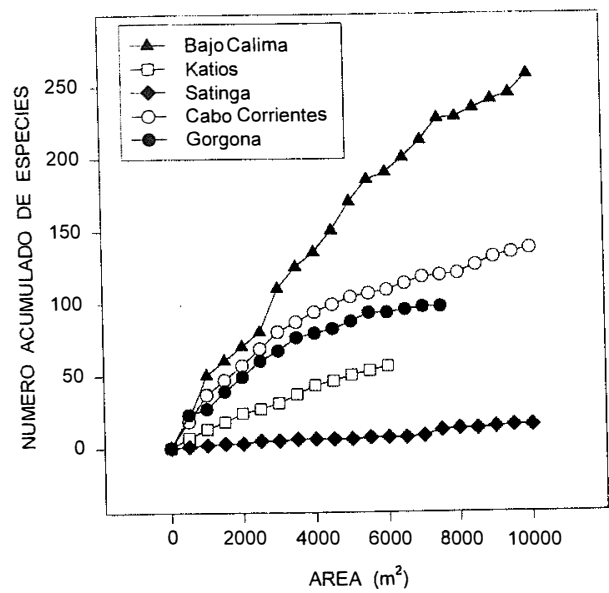

Fig. 1. Curvas de acumulación de especies de la Isla Gorgona, Cabo Corrientes (Galeano et al. 1993), Bajo Calima (FaberLangendoen \& Gentry 1991), Parque Los Katíos (Chocó) y Satinga (Nariño). Las curvas de la isla Gorgona, el Parque Los Katíos y Satinga fueron derivadas a partir de los censos realizados por Rangel (1990), Floresta (1984) y Codemaco (1973), respectivamente.

Indices de diversidad: Los bosques de Cabo Corrientes y de Bajo Calima obtuvieron valores del índice diversidad de Shannon (H) más altos (4.17 y 4.90 respectivamente) que para el bosque de la Isla Gorgona (3.92). Para el índice de Simpson, Bajo Calima presentó un valor más alto (63.1) que el de la isla Gorgona (36.3) y el de Cabo Corrientes (22.3). Para el índice de equitabilidad de Shannon, los bosques de la Isla Gorgona (0.86) y de Cabo Corrientes (0.85) presentaron valores muy similares, mientras que el bosque de Bajo Calima obtuvo un valor más alto (0.89). La isla Gorgona y el bosque de Bajo Calima presentaron valores más altos $(0.38$ y 0.27) para la equitabilidad de Simpson (E) que el bosque de Cabo Corrientes (0.16) (Cuadro 1).

\section{CUADRO 1}

Indices de riqueza y diversidad para los bosques de la isla Gorgona y del continente. El índice de Simpson (D) y su equitabilidad (E) se calcularon sólo para los bosques de la Isla Gorgona, de Cabo Corrientes y de Bajo Calima gracias a que se obtuvieron los censos originales de flora. Las áreas muestreadas fueron de 0.75 ha para la Isla Gorgona, 1.0 ha para el bosque de Cabo Corrientes y Bajo Calima, 0.1 ha para los bosques de Tutunendó y los del Ecuador.

\begin{tabular}{lll}
\multicolumn{2}{c}{ Shannon } & \multicolumn{2}{c}{ Simpson } \\
H $\quad$ J & D E E
\end{tabular}

Isla

$\begin{array}{lllll}\text { Isla Gorgona } \quad 3.92 & 0.86 & 36.3 & 0.38\end{array}$

Continente

Colombia

$\begin{array}{lllll}\text { Cabo Corrientes } & 4.17 & 0.85 & 22.3 & 0.16 \\ \text { Bajo Calima } & 4.90 & 0.89 & 63.1 & 0.27 \\ \text { Tutunendó } & 5.25 & 0.95 & & \end{array}$

Ecuador

$\begin{array}{lll}\text { Río Palenque 1 } & 4.26 & 0.89 \\ \text { Río Palenque 2 } & 4.28 & 0.89\end{array}$

Mecanismos de dispersión de frutos y semillas: Se encontró una proporción mayor de especies dispersadas por aves $(49.7 \%)$ y viento (6.7\%) en la Isla Gorgona que en el continente. En Cabo Corrientes un $30.2 \%$ de las especies fueron dispersadas por aves y un $4.5 \%$ dispersadas por viento y en Bajo Calima, un $22.3 \%$ de especies fueron dispersadas por aves y un $5.6 \%$ fueron dispersadas por viento. Los bosques continentales representados porCabo Corrientes y BajoCalima presentaron un porcentaje mayor de especies vegetales dispersadas por mamíferos no voladores (57.7\% y $63.6 \%$ respectivamente), arbóreos ( 30.2 $\%$ y $30.9 \%$ respectivamente) o terrestres $(27.5 \%$ y $30.9 \%$ respectivamente). El porcentaje de especies dispersadas por murciélagos fue muy similar para los tres bosques (8.6\% para Gorgona, $7.7 \%$ para Cabo Corrientes y $8.5 \%$ para Bajo Calima) (Fig. 2). 


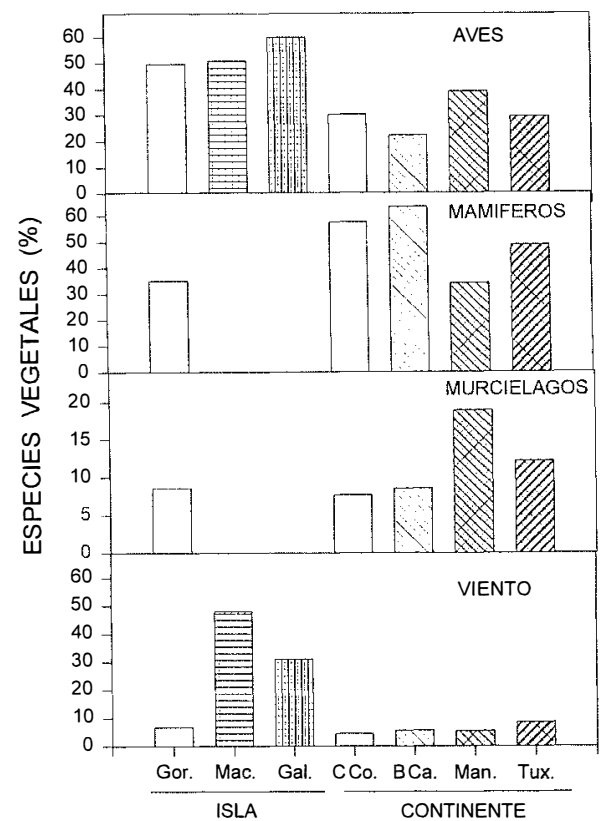

Fig. 2. Porcentaje de especies de plantas dispersadas por aves, mamíferos, murciélagos y viento en seis bosques neotropicales: Gor. $=$ Isla Gorgona, Mac.$=$ Serranía de Macuira, Gal.$=$ Islas Galápagos, Cco. $=$ Cabo Corrientes, $\mathrm{Bca} .=$ Bajo Calima, Man . = Manú y Tux. $=$ Los Tuxtlas. Los datos de dispersión se obtuvieron para tresislasbiogeográficas [Gorgona,La Macuira (Sugden 1982), y Galápagos (Porter 197.6)] y para cuatro bosques continentales de tierras bajas [Cabo Corrientes, Bajo Calima, el Parque Nacional Manú en el Perú (Foster et al. 1986) y el Parque Nacional Los Tuxtlas en México]. Los dispersorespotencialesparalasespeciesdeárboles deGorgona, Cabo Corrientes y Los Tuxtlas, fueron generados con base en la interpretación de las listas de plantas en Rangel (1990), Galeano et al. (1993) e Ibarra-Manríquez \& Oyama (1992), respectivamente. Los dispersores de las especies de Bajo Calima fueron generados con base en una lista de plantas proporcionada por Don Faber-Langendoen.

\section{DISCUSION}

Curvas de acumulación de especies: Las curvas muestran la baja riqueza de especies de la isla Gorgona al compararla con los bosques continentales del Chocóbiogeográficoreconocido por su alta diversidad (Gentry 1986). Este bajo número de especies puede ser el resultado de varios factores. $\mathrm{Al}$ aislarse hace 12000 años (Ortíz 1989), Gorgona debió soportar un mayor número de especies, el cual se vio reducido por los procesos de relajación y de extinción propios de islas continentales (Wilcox 1978). Además, su área $\left(14 \mathrm{~km}^{2}\right)$ limitaría el tamaño de las poblaciones, haciéndolas más susceptibles a la extinción(Diamond 1973). La distancia de la Isla al continente ( $32 \mathrm{~km}$ en línea recta) limitaría la migración de plantas dispersadas por mamíferos no voladores. Sin embargo, Wilcox (1978) encontróqueparaislascontinentalesestadistancia sería menos importante que los procesos de relajación y de extinción.

La riqueza de la isla Gorgona fue más alta que la del bosque del Parque Los Katíos, sometido à condicionesclimáticasmásrigurosas.EnelParque los Katíos hay una precipitación media anual de $1200 \mathrm{~mm}$ y un régimen de lluvias estacional, con una estación seca que va de diciembre a marzo. Este resultado es importante porque muestra que no todos los bosques de la región del Darién tienenriquezas y diversidadesaltas. De otra parte, el bosque de Satinga es un bosque de manglar que a pesar de tener condiciones climáticas similares a las de la Isla Gorgona, está sometido a inundaciones y salinidad, factores ambientales que limitan el número de especies. Es así como en este bosque la curva de acumulación de especies en función del área se saturó muy rápidamente.

Indices de diversidad: La diversidad de la isla Gorgona medida con el índice de Shannon (H) fue menor que la diversidad de los bosques del continente, lo cual apoya el resultado de las curvas de acumulación de especies que muestran una riqueza mayor de especies en los bosques del continente. El valor del índice de la isla, fue calculado para las especies encontradas en 7500 $\mathrm{m}^{2}$ mientras que el valor de Cabo Corrientes y Bajo Calima fueron calculados para $10000 \mathrm{~m}^{2}$. Estadiferenciaen el tamañodelamuestraafectaría el valor del índice sobrevaluando la riqueza de los bosques de Cabo Corrientes y Bajo Calima (Magurran 1988). Para eliminar esta posible duda sobre la baja riqueza de especies de la isla por el efecto del tamaño de la muestra, se comparó el 
valor de este índice con el de otras localidades en el Pacífico con un área de muestreo menor (1 000 $\mathrm{m}^{2}$ ) a la del censo de Cabo Corrientes $\left(10000 \mathrm{~m}^{2}\right)$. Se encontró que pese a las diferencias de tamaño del área de muestreo, en cinco bosques de la costa Pacífica, el índice de Shannon fue mayor que el de Gorgona. Por ejemplo, el índice de Shannon para el bosque de Tutunendó tuvo un valor de 5.25, seguido de Río Palenque 1 con 4.26 y Río Palenque 2 con 4.28 (Cuadro 1) (Alvarez 1993).

La equitabilidad de Shannon se encontró muy similar para los bosques de la isla Gorgona y de Cabo Corrientes. Este índice, que mide la razón entre la diversidad observada y la diversidad máxima(Magurran 1988), estuvo bastantecercano a 1 para la Isla Gorgona (0.86) y para Cabo Corrientes (0.85). Sin embargo, los otros cuatro bosques continentales del Pacífico incluyendo Bajo Calima, tuvieron un índice de equitabilidad de Shannon mayor que el de la isla Gorgona (Cuadro 1).Esto sugiere que la mayoría de especies en los bosques continentales poseen una cantidad similar de individuos, mientras que en la isla existen unas especies mejor representadas que otras.

El índice de Simpson (D) que mide tanto la dominancia como la riqueza, (Magurran 1988) tuvo un valor más alto para la isla(36.3), que para el bosque de CaboCorrientes(22.3). Esto muestra que la isla presenta un número mayor de especies dominantes que los bosques de Cabo Corrientes, lo cual apoya lo sugerido por el índice de equitabilidad de Shannon (Magurran 1988). La tala de árboles de madera fina en Cabo Corrientes (Galeano etal. 1993) pudooriginar la disminución del número de individuos de las especies más dominantes lo cual explica el valor inferior del índice de Simpson paraestebosque del continente. En el caso del bosque de Bajo Calima, el alto valor del índice de Simpson está indicando una gran riqueza de especies con respecto al bosque de la Isla Gorgona y la presencia de algunas especies dominantes.

Los valores obtenidos para el índice de equitabilidad de Simpson (E) en los bosques de
Gorgona, de Cabo Corrientes y de Bajo Calima son bajos lo cual evidencia la escasa uniformidad en la distribución de individuos entre las especies dominantes en estos bosques. No obstante, el valor de la equitabilidad de Simpson para el bosque de la Isla Gorgona (0.38) fue mayor que el del bosque de Cabo Corrientes (0.16) y el de Bajo Calima (0.27). Esto sugiere que los individuos de las especies dominantes de la isla presentan una distribución ligeramente más equitativa que los de los bosques continentales (Begon et al.1996).

Mecanismos de dispersión de frutos y semillas: La mayoría de plantas en Gorgona son dispersadas por aves, mientras que en Cabo Corrientes y en Bajo Calima son dispersadas por mamíferos (Fig. 2). Este resultado está de acuerdo a lo obtenido en otros bosques continentales tropicales de tierras bajas. En los Tuxtlas, parque nacional de México, los mamíferos no voladores (48.9\%) y las aves (29.5\%) son los dispersores más importantes (Ibarra-Manríquez \& Oyama 1992) al igual que en Cabo Corrientes (57.7 \% y $30.2 \%$, respectivamente) y en Bajo Calima (63.6 $\%$ y $22.3 \%$, respectivamente). En el Parque Nacional Manú (Perú), la dispersión por mamíferos terrestres es más alta que en la Isla Gorgona (Foster et al.1986) (Fig. 2). Las diferencias entre los bosques del continente y la isla Gorgona se pueden explicar por el obstáculo que representa el mar para la dispersión de animales terrestres. La llegada de mamíferos terrestres a la isla sólo sería posible por troncos de maderaarrojados al mar por los ríos que drenan la vertiente pacífica y que son llevados a la isla por las corrientes marinas. Un tronco tardaría en llegar a la isla Gorgona de 3.5 a 5 días, tiempo que probablemente causaría la deshidratación de un mamífero(Alberico 1986). El escaso número de mamíferos (4 especies) en la isla apoya esta idea (Alberico 1986). En bosques de tierras bajas, por el contrario, no hay barreras grandes que impidan el desplazamiento de mamíferos a través del bosque, por lo cual es un mecanismo de dispersión importante. Por su parte, las aves son capaces de atravesar los $32 \mathrm{~km}$ que separan a Gorgona del continente, lo que explicaría el alto porcentaje de dispersión por aves. La isla y los otros bosques 
tienen valores de dispersión por murciélagos muy similares. La migración de estos animales a Gorgona es factible por su habilidad de recorrer distancias cortas. En la islă, existen 14 especies de murciélagos (Alberico 1986) lo que sustenta esta interpretación.

Los resultados encontrados para otras islas biogeográficas o geográficas corroboran que la mayoría de plantas en este tipo de hábitats son dispersadas poraves. El bosque enano nublado de la Serranía de la Macuira es considerado como una isla por estar rodeado por bosques secos y espinosos. Sugden (1982)encontróque lamayoría de especies presentaban mecanismos de dispersión por aves $(51.0 \%)$ y por viento $(48.0 \%)$. De igual forma, en las islas Galápagos, el $60 \%$ de especies son dispersadas por aves y el $31 \%$ por viento (Porter 1976). La ausencia de mecanismos de dispersión por mamíferos, se explicaría porque las formaciones secas en el caso de Macuira, y el mar, en el caso de las isla Galápagos, constituyen grandes barreras para el paso de mamíferos continentales. Además, la distancia del bosque más cercano a la serranía y a las islas Galápagos es muy larga(200km y $1000 \mathrm{~km}$, respectivamente) (Pala 1981, Sugden 1982), lo cual dificultaría la llegada de murciélagosquienes tienen migraciones cortas (Sugden 1982). Laisla Gorgona no presenta este problema por estar separada del continente por sólo $32 \mathrm{~km}$. La historia antigua y reciente de Gorgona puede aclarar la presencia de plantas dispersadas por mamíferos. Por un lado, Alberico (1986) propone que la isla estuvo unida al continente durante las glaciaciones del Pleistoceno. La presencia de especies vegetales como Podocarpusoleifolius y Simaroubaamara que poseen semillas muy grandes para ser dispersadas por aves o murciélagos (mayores de $1 \mathrm{~cm}$ ), apoyan la idea de que hubo un posible desplazamiento de mamíferos terrestres durante este periodo. Esto podría explicar que haya un $21.6 \%$ de plantas dispersadas por mamíferos. Por otro lado, la introducción de especies en la isla por humanos, puede ser una de las razones por las cuales se encuentran especies normalmente dispersadas por mamíferos como por ejemplo
Inga edulis (Alberico 1986, Barbosa 1986).

En conclusión, el estudio comparativo de la riqueza y diversidad de plantas de los bosques de la Isla Gorgona y algunos de la costa pacífica colombo-ecuatoriana, permitieron concluir que los bosques de la isla son menos ricos y diversos. El mecanismo de dispersión más importante en la isla son las aves mientras que la dispersión por mamíferos es el mecanismo dominante en los bosques continentales de tierras bajas.

\section{AGRADECIMIENTOS}

Este trabajo se llevó a cabo gracias a la Fundación para la Promoción de la Investigación de la Ciencia y la Tecnología, Banco de la República, Colombia. Queremos dejar constancia de muestro agradecimiento a Luis G. Henao y Gloria Galeano(Fundación Inguedé, Bogotá) por facilitar los datos de los censos originales del bosque de Cabo Corrientes, a Don FaberLangendoen por facilitar la lista de especies de Bajo Calima, a Gilberto Mahecha por facilitar información sobre características de frutos y semillas de varios árboles y a Mery Santos por información sobre posibles dispersores de algunas de las especies de los bosques estudiados.

\section{RESUMEN}

En este trabajo se hizo una comparación entre la flora de la isla Gorgona (Colombia) y la de los bosques del Chocó biogeográfico (Colombia y Ecuador). Para esta comparación se utilizaron curvas de acumulación de especies e índices de riqueza y diversidad, tanto publicadas por diferentes autores o generadas a partir de los inventarios florísticos originales. La riqueza del bosque de la isla Gorgona calculada a partir de curvas especie-área y del índice de diversidad de Shannon fue inferior a la de varios bosques colombianos y ecuatorianos en las tierras bajas del continente. Adicionalmente, el índice de equitabilidad de Shannon en cinco bosqués del Pacífico colombo-ecuatoriano fue mayor que el de la isla Gorgona, lo cual sugiere que en los bosques continentales las especies están representadas por un número similar de individuós. $\mathrm{La}$ ornitocoria fue el mecanismo de dispersión más importante en 
la isla Gorgona ( $49.7 \%$ ), en forma similar a lo encontrado para las islas Galápagos en Ecuador $(60 \%)$ y para la isla biogeográfica del bosque nublado de la Serranía de la Macuira, Guajira, en el norte de Colombia (5I \%). En contraste, la dispersión por mamíferos fue el mecanismo más importante para las especies de árboles del bosque continental de Cabo Corrientes, de BajoCalima(Colombia) y los Tuxtlas(México). A pesar de que los bosques de la Isla Gorgona, no son especialmente ricos en especies, cumplen una importante función protectora de los ecosistemas de coral que rodean la isla. Una reducción de la cobertura boscosa tendría como consecuencia un aumento de las tasa de erosión y avalanchas que afectarían gravemente los ecosistemas marinos, en particular los arrecifes de coral.

\section{REFERENCIAS}

Alberico, M. 1986. Biogeografía terrestre, p.224-243. In H. von Prahl \& M. Alberico (ed.). Isla Gorgona. Banco Popular, Bogotá, Colombia.

Alvarez, E. 1993. Composición florística, diversidad, estructura, biomasade un bosque inundable en la Amazonía colombiana. Tesis de Magister, Universidad de Antioquia, Medellín, Colombia. 473 p.

Augspurger, C.K. 1986. Morphology and dispersal potential of wind-dispersed diaspores of neotropical trees. Amer. J. Bot. 73: 353-363.

Barbosa, C. 1986. Contribución al conocimiento de la flora y vegetación del Parque Nacional Natural isla Gorgona y Gorgonilla. Pérez- Arbelaezia 1: 311-335.

Bartholomäus, A., C.A. De la Rosa, G.J. Santos, L.E. Acero \& W. Moosbrugger. 1990. El manto de la tierra. CAR, Bogotá, Colombia. 332 p.

Begon, M., J. Harper \& C.R. Townsend. 1996. Ecology. Blackwell Scientific, Cambridge. 945 p.

Blake, J.G. \& B.A. Loiselle. 1992. Fruits in the diets of neotropical migrant birds in Costa Rica. Biotropica 24: 200-210.

Burrows, F.M. 1986. The aerial motion of seeds, fruits, spores and pollen, p. 1-48. In R. Murray (ed.). Seed dispersal. Academic, Sydney, Australia.

Codemaco S.A. 1973. Plan de ordenación Satinga, Sanquianga, Anexo 1. Inderena, Bogotá.

Connor, E.F. \& E.D. McCoy. 1979. The statictics and biology of the species-area relationship. Amer. Natur. 113: 791833.

Diamond, J. 1973. Distributional ecology of New Guinea birds. Science 179: 759-769.

Espinal, L. S. \& E. Montenegro. 1977. Zonas de vida o formaciones vegetales de Colombia. Memoria explicativa sobre el mapaecológico.IGAC, 13(11). Bogotá, Colombia. $238 \mathrm{p}$.

Faber-Langendoen, D. \& A.H. Gentry. 1991. The structure and diversity of rain forest at Bajo Calima, Chocó region, Western Colombia. Biotropica 23: 2-11.

Floresta Itda. 1984. Estudio ecológico de las asociaciones vegetales del Parque Nacional Natural Los Katíos. Inderena, Bogotá, Colombia. 220 p.

Foster, R.B., J.B. Arce \& T.S. Wachter. 1986. Dispersal and the sequential plant communities amazonian Perú foodplain, p. 357-370. In A. Estrada \& T.H. Fleming(ed). Frugivores and seeddispersal. Dr. Junk, La Haya, Holanda.

Galeano, G., J. Cediel, M. Pardo \& C. Hernández. 1993. Composición y diversidad de los bosques de la región del Cabo Corrientes, costa Pacífica del Chocó. Fundación Inguedé, Bogotá. 21 p.

Gansser, A. 1950. Geological and petrographic notes on Gorgona island in relation to North-Western South America. Schweiz, Mineral. Petrogr. 30: 219-237.

Gentry, A.H. 1986. Species richness and floristic composition of Chocó region plant communities. Caldasia 15: 72-91.

Gentry, A.H. 1993. A field guide to the families and genera of woody plants of Northwest South America (Colombia, Ecuador, Peru) with supplementary notes on herbaceous taxa. Conservation International, Washington. 895 p.

Haffer, J. 1970. Geologic-cl imatic history and zoogeographic significance of the Urabá region in Northwestern Colombia. Caldasia 10: 603-636.

Howe, H.F. 1984. Implications of seed dispersal by animal tropical reserve management. Biological Conservation 30: $261-281$.

Howe, H. \& L.C. Westley. 1988. Ecological relationships of plants and animals. Oxford University, Oxford. 273 p.

Ibarra-Manríquez, G. \& K. Oyama. 1992.Ecological correlates of reproductive traits of mexican rain forest trees. Amer. J. Bot. 79: 383-394.

Lack, D. 1969. The numbers of bird species on islands. Bird 
Stud. 16: 193-209.

Lack, D. 1976. Island biology, illustrated by the land birds of Jamaica. University of California, California. $445 \mathrm{p}$.

Lourenco, W.R. \& E. Flores. 1989. Los escorpiones (Chelicerata) de Colombia, la fauna de la isla Gorgona, aproximación biogeográfica. Caldasia 16: 66-70.

MacArthur, R.H. \& E.O. Wilson. 1967. The theory of island biogeography. Princenton University, New Jersey. 203 p.

Magurran, A. 1988.Ecological diversity and its measurement. Princenton University, New Jersey. 179 p.

Mori, S.A. \& J.L. Brown. 1994. Report on wind dispersal in a lowland moist forest in central French Guiana. Brittonia 46: $105-125$.

Nygren, W.E. 1950. Bolivar geosyncline of Northwestern South America. Bull. Amer. Ass. Petroleum Geol. 34: 1998-2006.

Ortiz, B. 1989. Caracterización biogeográfica y ecológica de las aves terrestres de la isla Gorgona. FEN, Bogotá, Colombia. $256 \mathrm{p}$.

Pala, S.A. 1981. Nueva enciclopedia Larousse. Tomo 5. Planeta, Barcelona España. 1063 p.

Porter, D.M. 1976. Geography and dispersal of Galápagos islands vascular plants. Nature 264: 745-746.

Prahl, H., F. Guhl \& M. Grögl. 1979. Gorgona. Futura Grupo, Bogotá, Colombia. 279 p.

Quinn, J.F. \& S.P. Harrison. 1988. Effects of habitat fragmentation and isolation on species richness: evidence from biogeographic patterns. Oecologia 75: 132-140.

Rangel, O. 1990. Tipos de vegetación, p. 109-126. In J. Aguirre \&O.Rangel (ed.). Biotay ecosistemas deGorgona. Fondo FEN, Bogotá, Colombia.

Rangel, O. \& J. Aguirre. 1993. La isla Gorgona y sus ecosistemas, p. 50-63. In P.Leyva(ed.).ColombiaPacífico. Fondo FEN, Bogotá, Colombia.

Renner, S. 1987. Seed dispersal, special topics. Progress in Botany 49: 413-432. Springer-Verlag, Berlín, Alemania.

Richman, A.D., T.J. Case \& T.D. Schwaner. 1988. Natural and unnatural extinction rates of reptiles on islands. Amer. Natur. 131: 611-630.

Sugden, A.M. 1982. Long distance dispersal, isolation, and the cloud forest flora of the serranía de Macuira, Guajira, Colombia. Biotropica 14: 208-219.

Valle, J.I. 1973. Introducción a la dendrología de Colombia. Universidad Nacional de Colombia, Medellín, Colombia. $550 \mathrm{p}$.

Wilcox, B.A. 1978. Supersaturated island faunas: a speciesage relationship for lizards on postpleistocene land-bridge island. Science 199: 996-998.

Williamson, M.H. 1981. Island population. Oxford University, Oxford. $286 \mathrm{p}$.

Zona, S.\& A. Henderson. 1989. A review of animal-mediated seed dispersal of palms. Selbyana 11:6-21. 